\title{
Influence de la fatigue sur les paramètres biomécaniques de la foulée mesurés par accéléromètrie
}

\section{Influence of fatigue on stride biomechanical parameters measured by an accelerometer}

\author{
Jidovtseff Boris ${ }^{1}$ \\ Rodriguez de la Cruz Carlos ${ }^{1}$ \\ Bury Thierry ${ }^{1}$, \\ Deflandre Dorian ${ }^{1}$ \\ ${ }^{1}$ Département des Sciences de la Motricité \\ Université de Liège \\ Jidovtseff Boris \\ ISEPK - B21 \\ Allée des Sports, 4 \\ $B-4000$ Liège \\ Belgique \\ E-mail : b.jidovtseff@uliege.be
}

\section{Résumé}

Objectifs. Cette étude a comme objectif principal d'explorer l'influence de la fatigue sur les paramètres biomécaniques de la foulée mesurés par un accéléromètre portatif, le MyotestRun. Le protocole mis sur pied a permis des analyses complémentaires sur la reproductibilité et la sensibilité de l'outil utilisé.

Méthode. Trente-deux sujets masculins, répartis en deux groupes (COUREURS et CONTROLE), ont réalisé un test d'effort progressif sur tapis roulant, des séquences de course à vitesse standardisée sur piste d'athlétisme et un test de temps limite à 90\% de la VMA sur piste. Les mesures biomécaniques obtenues à partir du MyotestRun ont permis d'explorer la reproductibilité des données, l'influence de la vitesse, les différences entre course sur tapis roulant et course sur piste ainsi que l'influence de la fatigue sur les paramètres biomécaniques

Résultats. Les résultats montrent une excellente reproductibilité inter-essais des paramètres du MyotestRun à l'exception de l'asymétrie de la course. Aucune différence significative n'apparaît entre la foulée sur tapis roulant et la foulée sur piste. Le test progressif montre que tous les paramètres évoluent avec l'augmentation de la vitesse, confirmant la sensibilité du MyotestRun. L'étude met en évidence une modification de la foulée avec la fatigue qui se caractérise par un allongement du temps d'appui au sol et une diminution de la force, sans altération de la fréquence. Si l'étude met en évidence que l'évolution de la foulée avec la fatigue est plus uniforme 
dans le groupe COUREURS elle souligne également des adaptations à la fatigue qui peuvent être très différentes d'un sujet à l'autre.

Conclusion. Cette étude démontre d'une part, que le MyotestRun peut être un outil de terrain utile à l'analyse biomécanique de la course, et d'autre part, que l'influence de la fatigue sur la foulée, s'accorde avec la plupart des études tout en confirmant des différences interindividuelles très importantes.

Mots-clés : foulée ; course ; fatigue ; biomécanique ; myotest ; reproductibilité

\section{Abstract}

Aims of the study. The aim of the study was to investigate the influence of fatigue on stride biomechanics measured by a portable accelerometer, the MyotestRun. Opportunities of the protocol allowed additional analysis on the device reliability and sensitivity.

Methods. Thirty-two male subjects, divided in two groups (runners and control), were involved in the study and achieved a treadmill progressive VO2max test; repeated run at constant velocity on a track and a field test at $90 \%$ of the VvO2max until exhaustion. The biomechanical measurements obtained from the MyotestRun allow exploring the reliability, the influence of speed, the differences between treadmill and track stride and the influence of fatigue.

Results. Results show an excellent reproducibility of the MyotestRun values, except for the asymmetry of the stride. No significant stride difference appears between treadmill and track conditions. The progressive test shows that all the parameters evolve with speed increment, confirming the sensitivity of the MyotestRun. The study shows changes in the stride with the fatigue, such as an increase of the contact time, a decrease of the force. However any change in the stride frequency is observed. If the study shows that the fatigue pattern is more constant in the runners group it also highlights adaptations to fatigue that can be very different from one subject to another.

Conclusion. This study confirmed that MyotestRun is a useful device in the stride analysis. The influence of fatigue on the stride is in accordance with most of the studies while confirming very important interindividual differences.

Key-words : stride ; run ; fatigue ; biomechanics ; myotest ; reproducibility

\section{Introduction}

La course à pied apparaît comme une activité physique simple, naturelle, accessible à tous et connaît en engouement croissant caractérisé par une multiplication du nombre de courses organisées ainsi qu'une augmentation de leur fréquentation. Quel que soit le niveau sportif, l'analyse de la foulée apparaît particulièrement intéressant car elle présente un impact sur la performance (1-3), mais aussi sur les risques de blessure [4-7].

L'exploration biomécanique de la foulée, nécessite généralement un matériel sophistiqué, peu accessible et au traitement complexe comme les plateformes de force, les analyses tridimensionnelles ou encore des stations inertielles embarquées. Ces approches, onéreuses et chronophages restent le privilège des scientifiques et des élites sportives. Bien que l'œil expérimenté d'un entraîneur permette d'identifier une série de problèmes techniques il ne permet en aucun cas de quantifier les paramètres de la foulée. A l'heure actuelle, les outils d'analyse biomécanique de la foulée accessibles au large public restent limités. Les microtechnologies, basées sur l'accélérométrie et le géo-positionnement par satellite (GPS), apparaissent prometteuses car elles permettent d'étudier sur le terrain une série de 
paramètres biomécaniques intéressants comme la fréquence et la durée des appuis ou encore l'estimation de la vitesse [8-15]. La concurrence commerciale sur ces outils technologiques conduit à une réduction des prix et facilite à l'accès à certains paramètres biomécaniques. Les possibilités d'analyse restent limitées et dans la plupart des cas, les paramètres proposés n'ont fait l'objet d'aucune vérification scientifique. Dans ce paysage des nouvelles technologies utiles à l'entraînement, le MyotestRun (Myotest SA, Suisse) s'avère être un outil extrêmement léger, utilisable sur le terrain et permettant la quantification d'un grand nombre de paramètres biomécaniques de la foulée. Une publication récente s'est intéressée à cet outil en étudiant la reproductibilité inter-essais et la validité de trois paramètres importants de la foulée : la fréquence (Fr), le temps d'appui sol (TA) et le temps de vol (TV) [10]. La comparaison du MyotestRun à deux autres systèmes de références, à savoir l'Optojump Next et la caméra haute vitesse, démontre l'excellente validité de la fréquence. La mesure du temps de contact par le MyotestRun était par contre raccourcie de $34 \%$ alors que la mesure du temps de suspension était allongée en moyenne de 64\% [10)]. Ces différences très importantes s'expliquent par le fait que l'identification des phases de vol et d'appui diffère en fonction de l'outil utilisé. Avec l'Optojump et avec la caméra haute vitesse, la division de la foulée en phase aérienne et phase de contact se fait en repérant les moments où le pied entre en contact et quitte le sol. Avec le MyotestRun ces deux phases sont identifiées en repérant la période pendant laquelle la force verticale est supérieure (TA) ou inférieur (TV) au poids de l'individu. Le temps de contact ainsi mesuré correspond en réalité au temps de contact effectif décrit par Cavagna et al [16)]. II s'agit donc de deux approches différentes qui ne peuvent pas être directement comparées et il importe avant tout pour les entraîneurs et scientifiques de connaître les différences entre les deux approches. Gindre et al [10] proposent une équation permettant de retrouver les valeurs de TA et TV à partir du MyotestRun qui seraient comparables à celles de l'Optojump et de la caméra haute vitesse. Une autre étude, réalisée par Goutebarge et al [17], confirme la bonne validité des mesures de fréquence ainsi que les différences observées pour le temps d'appui. Elle pemet par contre en évidence une faible reproductibilité inter-séance de TA ce qui est en contradiction avec l'étude de Gindre et al [10] qui, dans une approche méthodologique différente, montre que les trois paramètres du MyotestRun étudiés possèdent une bonne reproductibilité inter-essais avec des coefficients de corrélation intraclasse $(\mathrm{CCl}) \geq 0,82$ et des coefficients de variation inférieurs à $10 \%$. Des études complémentaires apparaissent nécessaires afin de vérifier si le MyotestRun peut être considéré comme un outil de terrain pertinent utilisable à des fins scientifiques mais aussi d'entraînement. Les perspectives scientifiques les plus intéressantes sont d'une part l'analyse du pattern de foulée individuel et d'autre part l'évolution des paramètres de la foulée avec l'apparition de la fatigue. En effet, de nombreuses études aux protocoles très variables ont mis en évidence que la foulée se modifiait avec l'état de fatigue [18-21]. Ainsi à titre d'exemple, l'étude de d'Elliot et Ackland [18] montre qu'au cours d'un $10000 \mathrm{~m}$, la fatigue s'accompagne d'une réduction significative de la vitesse et de l'amplitude de la foulée. Candau et al [19] montrent, quant à eux, que lors d'un effort à intensité constante poussé jusqu'à l'épuisement, on observe une augmentation de la fréquences et de la variabilité des appuis et du coût énergétique de la course [19]. L'ensemble de ces travaux utilise un équipement de laboratoire complexe, couteux, et qui rend impossible les mesures sur le terrain. De par sa portabilité et son 
accessibilité le MyotestRun apparait comme une alternative intéressante dans ce type d'étude.

L'objectif principal de cette recherche est donc d'explorer sur le terrain à partir du MyotestRun l'influence de la fatigue sur les paramètres biomécaniques de la foulée. Toutefois, au vue des divergences apparues dans la littérature et afin de s'assurer de l'intérêt de notre démarche scientifique le protocole mis sur pied a permis l'exploration complémentaire de la reproductibilité et la sensibilité du MyotestRun. Une analyse préliminaire portera donc sur les trois questions suivantes : 1) Quel est le niveau de reproductibilité inter-essais des paramètres biomécaniques du MyotestRun ? 2) Est-ce que les paramètres biomécaniques observés à une même vitesse sont les mêmes sur tapis roulant et sur piste ? 3) Est-ce que les paramètres biomécaniques du MyotestRun évoluent avec la vitesse de course et est-ce que les valeurs observées sont conformes à la littérature ? Sur base des résultats observés les paramètres biomécaniques présentant un intérêt potentiel seront conservés afin d'analyser l'influence de la fatigue sur la biomécanique de la foulée chez des coureurs réguliers et non réguliers.

\section{Matériel et méthode}

\section{Sujets}

Trente-deux sujets masculins volontaires (âge $=23,8 \pm 4,6$ ans $;$ taille $=179,3 \pm 5,6 \mathrm{~cm}$; masse $=$ $71,5 \pm 10,5 \mathrm{~kg}$, vitesse maximale aérobie $(\mathrm{VMA})=16,8 \pm 2,1 \mathrm{~km} / \mathrm{h}$ ) ont participé au protocole. Parmi ceux-ci, 18 sujets (âge $=23,6 \pm 5,8 \mathrm{ans}$; taille $=177 \pm 5 \mathrm{~cm}$; masse $=67 \pm 7,3 \mathrm{~kg} ; \mathrm{VMA}=18,4 \pm 0,9 \mathrm{~km} / \mathrm{h}$ ) pratiquaient la course à pied à raison d'au minimum trois séances par semaine et avaient l'habitude des courses prolongées à intensité élevée (groupe COUREURS) et 12 sujets (âge $=24,25 \pm 2$,3ans ; taille $=182 \pm 5 \mathrm{~cm} ;$ masse $=79,1 \pm 11,1 \mathrm{~kg} ; \mathrm{VMA}=14,4 \pm 0,5 \mathrm{~km} / \mathrm{h}$ ) étaient des sportifs de loisir réguliers mais ne pratiquant pas la course à pied de manière régulière (moins de deux fois par semaine) (groupe CONTROLE). Tous les sujets retenus dans l'étude devaient avoir une VMA supérieure ou égale à $14 \mathrm{~km} / \mathrm{h}$.

\section{Protocole expérimental}

La première épreuve consistait à réaliser un test maximal progressif sur tapis roulant. La pente était de $1 \%$ et la température ambiante moyenne du laboratoire était de $21 \pm 1^{\circ} \mathrm{C}$. Après différentes mesures morphologiques, la pose du masque permettant la mesure des échanges gazeux et l'installation du MyotestRun au niveau abdominal, les sujets étaient invités à s'échauffer pendant cinq minutes en courant sur un tapis roulant à $8 \mathrm{~km} / \mathrm{h}$. La vitesse était augmentée de $2 \mathrm{~km} / \mathrm{h}$ toutes les trois minutes jusque 14 ou $16 \mathrm{~km} / \mathrm{h}$ en fonction du niveau du coureur. Ensuite, l'incrément de la vitesse était de $1 \mathrm{~km} / \mathrm{h}$ toutes les 3 minutes jusqu'à l'arrêt de l'effort. A chaque palier, après une minute de course à allure constante, les paramètres biomécaniques de la foulée étaient enregistrés pendant vingt secondes par le MyotestRun. Au signal de l'expérimentateur, le sujet appuyait sur le bouton central de l'accéléromètre pour déclencher l'enregistrement, et répétait la procédure 20 secondes plus tard afin d'arrêter l'enregistrement. 
Durant l'épreuve d'effort, les gaz respiratoires (VE, VO2, VCO2) étaient enregistrés de manière continue par un analyseur des gaz respiratoires (SensorMedics Vmax 29C, Italie). La fréquence cardiaque était mesurée à partir d'un cardio fréquencemètre (Polar S610, Finlande). De plus, à la fin de chaque palier et en fin de test, un prélèvement sanguin fut réalisé à l'index ou au majeur de la main droite des participants afin de doser la lactatémie par un analyseur automatique (YSI 1500 Sport L-Lactate Analyser, USA). Le test était arrêté par l'expérimentateur lorsqu'un des critères suivants était observés : a) épuisement du sujet ; b) quotient respiratoire > 1,10 ; c) plafonnement de la VO2 ; d) concentration en lactate $>8 \mathrm{mmol} / \mathrm{L}$. La VMA mesurée au terme de cette épreuve était retenue pour déterminer la vitesse de chaque sujet lors de l'épreuve à intensité constante réalisée lors de la deuxième session (90\% VMA).

La deuxième épreuve se déroulait sur une piste d'athlétisme entre une et quatre semaines après la première épreuve, en fonction de la disponibilité de l'athlète, de l'expérimentateur et des conditions climatiques. Après un échauffement standardisé et l'installation du MyotestRun, les sujets ont réalisé plusieurs enregistrements de la foulée sur une ligne droite de $100 \mathrm{~m}$. Deux vitesses ont été retenues: $10 \mathrm{~km} / \mathrm{h}$ et $90 \%$ VMA. Afin que le sujet respecte scrupuleusement les deux vitesses imposées, nous avons placé sur la ligne droite des cônes à la distance correspondant à trois seconde de course à l'allure déterminée. La reproductibilité inter-essai du MyotestRun a été vérifiée pour ces deux vitesses de course, par trois enregistrements de la foulée. L'épreuve principale consistait à maintenir le plus longtemps possible un effort à allure constante fixée à $90 \%$ de la VMA. Les sujets étaient équipés d'un cardio-fréquencemètre (Polar S610, Finlande) et du MyotestRun fixé à la ceinture. Afin de respecter scrupuleusement l'allure demandée, une montre était programmée pour bipper tous les $100 \mathrm{~m}$. A chaque tour de piste, il était demandé au sujet d'enregistrer les paramètres biomécaniques de la foulée en appuyant sur le bouton central du MyotestRun au début et à la fin d'une zone de 80 mètres située dans la ligne droite d'arrivée de la piste d'athlétisme. Tout au long de l'effort, les participants étaient vivement encouragés afin de bien respecter le rythme et d'aller le plus loin possible dans l'effort.

\section{Matériel}

Le MyotestRun est un accéléromètre triaxial portatif sans fil qui, lors de l'analyse de la course, est fixé sur une ceinture élastique entre le pubis et l'ombilic, conformément aux recommandations du constructeur. Les signaux sont enregistrés à une fréquence de $500 \mathrm{hz}$. II possède un micrologiciel interne qui permet d'analyser la foulée lors de la course. Le traitement du signal est basé sur plusieurs données qui doivent être soit mesurées, soit introduites dans le MyotestRun par l'expérimentateur ; L'accélération dans les trois axes est mesurée par le capteur tandis que la masse, la taille et la vitesse de course sont introduits dans le micrologiciel. La course doit être analysée en ligne droite et à allure constante. L'enregistrement est initié par le coureur lui-même en appuyant sur le bouton central du MyotestRun et se termine en appuyant une deuxième fois sur ce même bouton. Un bip auditif permet de distinguer le début de l'enregistrement de la fin. Le MyotestRun offre la possibilité d'analyser la 
foulée soit sur une distance déterminée, soit à une vitesse constante. Etant donné qu'il existe parfois un petit décalage de temps entre la pression du bouton central et le déclenchement de l'enregistrement, il a été décidé dans le cadre de cette étude d'introduire la vitesse de course qui doit être maintenue constante tout au long de l'épreuve.

Le micrologiciel MyotestRun offre douze paramètres biomécaniques dignes d'intérêt calculés selon les bases suivantes :

1. La fréquence des pas (FR, en pas/min) qui correspond au nombre de pas par minute. Ce paramètre est calculé sur base de la fréquence ondulatoire du signal accélération.

2. Longueur des pas (LG, en $\mathrm{m}$ ) qui est la distance moyenne entre deux appuis successifs. Elle est calculée par le rapport entre la distance parcourue durant l'enregistrement et le nombre de pas mesurés. La distance parcourue étant obtenue par le produit de la vitesse et du temps. Le nombre de pas est compté directement à partir du signal ondulatoire et correspond au produit de FR et du temps d'enregistrement.

3. Le temps d'appui effectif (TA, en s) correspond à la durée moyenne du contact du pied avec le sol pendant lequel la force exercée par le coureur sur le sol est supérieure ou égale à son poids [16].

4. Le temps de vol (TV, en s) est le temps passé en l'air et est identifié lorsque la force exercée par le coureur est inférieure à son poids. Ces deux mesures de temps sont calculées sur base de l'analyse du signal accélération.

5. L'indice de réactivité (REA) est le rapport entre TV et TA.

6. La distance d'appui (DA, en $\mathrm{m}$ ) correspond à la distance moyenne parcourue par le bassin pendant TA. DA est obtenu par le produit de la vitesse de course et de TA.

7. La force $(F$, en $N / \mathrm{kg})$ correspond à la composante verticale de la force par unité de masse

8. appliquée en moyenne au sol par le coureur durant l'appui. Elle est directement obtenue à partir du signal accélération du capteur.

9. Le déplacement vertical ( $D V$, en $\mathrm{m}$ ) est la distance moyenne entre le point le plus haut et le point le plus bas du MyotestRun lors d'un pas. Cette distance verticale est calculée par double intégration de l'accélération verticale.

10. La raideur ( $R$ en $\mathrm{kN} / \mathrm{m}$ ) est le rapport entre $\mathrm{F}$ et l'abaissement vertical moyen mesuré durant l'appui.

11. L'angle de pose de la jambe (APJ, en ${ }^{\circ}$ ) est l'angle formé par le sol et la jambe qui entame l'appui. Cet angle est calculé à partir de DA, de l'identification temporelle de l'abaissement vertical et de la taille des jambes estimée sur base de la taille du sujet.

12. Angle de décollage du centre de gravité $\left(A D\right.$ en $\left.{ }^{\circ}\right)$ est l'angle formé entre l'horizontale et la trajectoire du CG en fin de poussée. II est calculé à partir de DV et la vitesse de course.

13. L'indice de régularité (IR en \%) offre un aperçu en pourcent de la reproductibilité tout au long de l'enregistrement du ratio entre le TV et TA. 


\section{Analyse statistique}

Des statistiques descriptives basées sur la moyenne et l'écart-type ont été réalisées afin de présenter les valeurs de toute la population dans les différentes conditions d'effort et pour chaque paramètre. Un test de Shapiro-Wilk a permis de vérifier la distribution normale des données. Des tests paramétriques et non paramétriques ont été sélectionnés en fonction des résultats observés. Pour les comparaisons de deux échantillons provenant d'un même groupe un test de Student apparié et un test de Wilcoxon ont été utilisés. Pour les comparaisons de deux groupes différents, un test de Student non apparié et un test de Mann-Whitney ont été utilisés. L'analyse de mesures répétées est réalisée à partir d'un ANOVA pour mesures répétées classique ou d'une ANOVA de Friedman lorsqu'un test non paramétrique doit être utilisé.

La reproductibilité inter-séance des paramètres du MyotestRun a été étudiée en comparant les trois enregistrements réalisés pour une même vitesse standardisée. Deux vitesses différentes ont été étudiées lors de l'évaluation sur piste : $10 \mathrm{~km} / \mathrm{h}$ et $90 \%$ de la VMA. Trois outils statistiques ont été utilisés : le coefficient de variation inter-essais (CV, en \%), le coefficient de corrélation intra-classe (CCI) et l'erreur type de mesure (ETM). Les ICC obtenus pour la reproductibilité sont interprétés comme bon pour $I C C>0,75$; moyen pour $0,50 \leq I C C \leq 0,75$, et faible pour $I C C<0,50$ [21]. Les mêmes outils statistiques ont été utilisés pour comparer la foulée sur tapis roulant à la foulée sur piste à $10 \mathrm{~km} / \mathrm{h}$, Les statistiques ont été réalisées sur les logiciels Excel (Miscrosoft, USA) et Statistica (Statsoft, France).

\section{Résultats}

a) Reproductibilité inter-essais

La reproductibilité des paramètres du MyotestRun est présentée dans le Tableau 1. II ressort qu'aussi bien à $10 \mathrm{~km} / \mathrm{h}$ qu'à $90 \%$ de la VMA les CV de tous les paramètres sont inférieurs à $5 \%$. L'erreur type de mesure apparaît faible, atteignant au maximum $6,4 \%$ de la valeur moyenne pour $\mathrm{R}$ à $90 \%$ de VMA. Tous les paramètres montrent d'excellentes valeurs pour le $\mathrm{CCl}$ à l'exception de l'index de régularité à $10 \mathrm{~km} / \mathrm{h}(\mathrm{r}=0,53)$ et à $90 \%$ de la VMA $(r=0,39)$.

b) Comparaison tapis roulant - piste

La comparaison entre les résultats obtenus à $10 \mathrm{~km} / \mathrm{h}$ sur piste avec ceux obtenus à la même vitesse sur tapis roulant montre qu'il n'existe aucune différence significative entre les deux modalités de course $(p>0,05)$. L'analyse des paramètres de reproductibilité (CV, CCl et ETM) montre cependant une plus grande variabilité des paramètres comparativement avec la reproductibilité inter-essais réalisée à $10 \mathrm{~km} / \mathrm{h}$ sur piste (Tableau 1). À l'exception de l'index de régularité, les ICC sont compris entre 0,63 et 0,81 et sont considérés comme moyens à bons.

c) Influence de la vitesse

Le Tableau 2 reprend l'évolution de chaque paramètre aux quatre vitesses réalisées par tous les sujets à savoir $8,10,12 \mathrm{~km} / \mathrm{h}$ ainsi qu'à la VMA. L'analyse montre pour l'ensemble des sujets une influence de la vitesse hautement significative pour tous les paramètres à l'exception de TV qui ne se 
modifie pas avec la vitesse. La comparaison entre les deux sous-groupes montre que les coureurs présentent une fréquence cardiaque et une lactatémie inférieures aux trois premières vitesses mais pas à la VMA. La raideur (R) apparaît plus élevée chez les non coureurs à 10 et $12 \mathrm{~km} / \mathrm{h}$ comparativement aux coureurs. A VMA, le déplacement sur l'appui (DA) apparaît plus faible dans le groupe CONTROLE alors que les paramètres AD, APJ et DV s'avèrent supérieurs en comparaison avec le groupe COUREURS.

Tableau 1 : Valeurs moyennes (écarts-type) des paramètres du MyotestRun dans trois conditions (10km/h sur piste ; $90 \%$ VMA sur piste et $10 \mathrm{~km} / \mathrm{h}$ sur tapis roulant). Analyse de la reproductibilité inter-essais sur piste à $10 \mathrm{~km} / \mathrm{h}$ et à $90 \%$ de la VMA à partir variation du coefficient de variation (CV, en \%), du coefficient de corrélation intraclasse (CCI) et de l'erreur type de mesure (ETM). Analyse comparative entre la course sur tapis roulant et la course sur piste à $10 \mathrm{~km} / \mathrm{h}$ sur base des $\mathrm{CV}, \mathrm{CCl}$ et ETM.

\begin{tabular}{|c|c|c|c|c|c|c|c|c|c|c|c|c|c|}
\hline & & $\begin{array}{c}\mathrm{FR} \\
\text { (pas/min) }\end{array}$ & $\begin{array}{l}\text { LG } \\
(\mathrm{m})\end{array}$ & $\begin{array}{l}\text { TA } \\
\text { (s) }\end{array}$ & $\begin{array}{l}\text { TV } \\
\text { (s) }\end{array}$ & $\begin{array}{l}\text { DA } \\
\text { (s) }\end{array}$ & $\begin{array}{l}\text { AD } \\
\left({ }^{\circ}\right)\end{array}$ & $\begin{array}{l}\text { APJ } \\
\left({ }^{\circ}\right)\end{array}$ & $\begin{array}{l}\text { DV } \\
(\mathrm{m})\end{array}$ & $\begin{array}{c}R \\
(k N / m)\end{array}$ & REA & $\begin{array}{c}\mathbf{F} \\
(\mathrm{N} / \mathrm{kg})\end{array}$ & IR (\%) \\
\hline \multicolumn{14}{|c|}{ Reproductibilité inter-essais } \\
\hline \multirow{4}{*}{$\begin{array}{c}\text { Piste } \\
10 \mathrm{~km} / \mathrm{h}\end{array}$} & Moyenne & $160(8)$ & $1,05(0,05)$ & $0,175(0,011)$ & $0,202(0,015)$ & $0,49(0,03)$ & $19,6(1,4)$ & $75,6(1,2)$ & $0,115(0,013)$ & $36,5(5,1)$ & $1,15(0,11)$ & $19,2(1,5)$ & $93(3)$ \\
\hline & CV (\%) & 0,8 & 0,9 & 1,8 & 2,1 & 2,1 & 2 & 0,4 & 2,1 & 2,8 & 4,6 & 2,6 & 3,3 \\
\hline & $\mathrm{CCl}$ & 0,98 & 0,98 & 0,92 & 0.93 & 0,89 & 0,93 & 0,88 & 0,96 & 0,96 & 0,88 & 0,91 & 0,53 \\
\hline & ETM & 1,7 & 0,01 & 0,004 & 0,005 & 0,01 & 0,45 & 0,34 & 0,003 & 1,3 & 0,07 & 0,6 & 4,2 \\
\hline \multirow{4}{*}{$\begin{array}{c}\text { Piste } \\
90 \% \text { VMA }\end{array}$} & Moyenne & $169(10)$ & $1,50(0,15)$ & $0,151(0,017)$ & $0,205(0,014)$ & $0,63(0,06)$ & $13,6(1,7)$ & $70,9(1,7)$ & $0,108(0,012)$ & $47,9(8,9)$ & $1,38(0,19)$ & $22,1(2,2)$ & $92(2)$ \\
\hline & CV (\%) & 1,2 & 1,2 & 2,4 & 1,5 & 2,5 & 1,5 & 0,7 & 2,2 & 4,2 & 3,7 & 1,8 & 3,3 \\
\hline & $\mathrm{CCl}$ & 0,96 & 0,99 & 0,96 & 0.96 & 0,93 & 0,99 & 0,93 & 0,96 & 0,95 & 0,96 & 0,97 & 0,39 \\
\hline & ETM & 2,8 & 0,02 & 0,005 & 0,004 & 0,002 & 0,28 & 0,65 & 0,003 & 3,1 & 0,07 & 0,6 & 4,1 \\
\hline \multicolumn{14}{|c|}{ Analyse comparative entre la course sur tapis roulant et la course sur piste } \\
\hline Tapis roulant & Moyenne & 161(8) & $1,04(0,05)$ & $0,172(0,015)$ & $0,203(0,018)$ & $0,48(0,4)$ & $19,6(1,4)$ & $75,3(0,8)$ & $0,116(0,013)$ & $38,2(8,3)$ & $1,20(0,21)$ & $19,2(2,3)$ & $94(3)$ \\
\hline \multirow{3}{*}{$10 \mathrm{Km} / \mathrm{h}$} & CV $(\%)$ & 2,4 & 2,5 & 5,3 & 4,7 & 5,2 & 4,6 & 1,0 & 5,9 & 8,4 & 9,5 & 6,7 & 7,2 \\
\hline & $\mathrm{CCl}$ & 0,82 & 0,81 & 0,74 & 0.73 & 0,75 & 0,74 & 0,67 & 0,78 & 0,76 & 0,63 & 0,71 & 0,53 \\
\hline & ETM & 3,8 & 0,03 & 0,008 & 0,010 & 0,02 & 0,88 & 0,69 & 0,007 & 3,8 & 0,12 & 1,2 & 5,8 \\
\hline
\end{tabular}

Tableau 2 : évolution des paramètres de la course en fonction de la vitesse au cours de l'épreuve progressive sur tapis roulant. L'influence de la vitesse sur chaque paramètre est représentée par la $p$ value sur la dernière ligne. Les différences entre les deux groupes pour un même paramètre à une même vitesse sont représentées par * pour p<0,05; ${ }^{* *}$ pour $p<0,01$ et ${ }^{* * *}$ pour $p<0,001$.

\begin{tabular}{|c|c|c|c|c|c|c|c|c|c|c|c|c|c|c|}
\hline & & $\begin{array}{c}F C \\
(b m p)\end{array}$ & $\begin{array}{c}L A \\
(\mathrm{mmole} / \mathrm{l})\end{array}$ & $\begin{array}{c}F R \\
\text { (pas/min) }\end{array}$ & $\begin{array}{l}L G \\
(m)\end{array}$ & $\begin{array}{l}T A \\
(s)\end{array}$ & $\begin{array}{l}T V \\
(s)\end{array}$ & $\begin{array}{l}D A \\
(m)\end{array}$ & $\begin{array}{l}A D \\
\left(0^{\circ}\right)\end{array}$ & $\begin{array}{l}A P J \\
\left({ }^{\circ}\right)\end{array}$ & $\begin{array}{l}D V \\
(m)\end{array}$ & $\begin{array}{c}R \\
(k N / m)\end{array}$ & $R E A$ & $\begin{array}{c}F \\
(N / k g)\end{array}$ \\
\hline \multirow{4}{*}{ Coureurs } & $8 \mathrm{~km} / \mathrm{h}$ & $133 \pm 13$ & $1,2 \pm 0,4$ & $157 \pm 11$ & $0,85 \pm 0,07$ & $0,184 \pm 0,023$ & $0,200 \pm 0,016$ & $0,41 \pm 0,05$ & $23,8 \pm 1,6$ & $77,4 \pm 1,7$ & $0,12 \pm 0,02$ & $32 \pm 6$ & $1,09 \pm 0,13$ & $18,0 \pm 1,6$ \\
\hline & $10 \mathrm{~km} / \mathrm{h}$ & $145 \pm 15$ & $1,4 \pm 0,6$ & $161 \pm 9$ & $1,04 \pm 0,06$ & $0,172 \pm 0,011$ & $0,202 \pm 0,015$ & $0,48 \pm 0,03$ & $19,6 \pm 1,4$ & $75,5 \pm 0,8$ & $0,11 \pm 0,01$ & $35 \pm 5$ & $1,18 \pm 0,12$ & $19,2 \pm 1,6$ \\
\hline & $12 \mathrm{~km} / \mathrm{h}$ & $160 \pm 16$ & $1,7 \pm 0,7$ & $164 \pm 8$ & $1,22 \pm 0,06$ & $0,164 \pm 0,012$ & $0,203 \pm 0,013$ & $0,55 \pm 0,04$ & $16,6 \pm 1,0$ & $73,5 \pm 0,9$ & $0,11 \pm 0,01$ & $38 \pm 6$ & $1,24 \pm 0,13$ & $20,1 \pm 1,6$ \\
\hline & VMA & $197 \pm 8$ & $8,1 \pm 1,7$ & $180 \pm 12$ & $1,70 \pm 0,11$ & $0,138 \pm 0,018$ & $0,196 \pm 0,018$ & $0,70 \pm 0,10$ & $10,7 \pm 1,2$ & $69,3 \pm 2,5$ & $0,10 \pm 0,01$ & $54 \pm 13$ & $1,44 \pm 0,29$ & $22,3 \pm 2,7$ \\
\hline \multirow{4}{*}{ Contrôle } & $8 \mathrm{~km} / \mathrm{h}$ & $\underset{* *}{151 \pm 13}$ & $\begin{array}{c}1,9 \pm 0,5 \\
* * *\end{array}$ & $148 \pm 21$ & $0,92 \pm 0,17$ & $0,189 \pm 0,041$ & $0,227 \pm 0,057$ & $0,42 \pm 0,09$ & $26,3 \pm 5,1$ & $77,5 \pm 2,6$ & $0,15 \pm 0,07$ & $37 \pm 12$ & $1,23 \pm 0,35$ & $17,3 \pm 2,5$ \\
\hline & $10 \mathrm{~km} / \mathrm{h}$ & $\underset{* * *}{167 \pm 11}$ & $\begin{array}{c}2,5 \pm 0,5 \\
* * *\end{array}$ & $159 \pm 7$ & $1,05 \pm 0,05$ & $0,172 \pm 0,021$ & $0,206 \pm 0,022$ & $0,48 \pm 0,06$ & $20,0 \pm 1,9$ & $75,9 \pm 1,7$ & $0,12 \pm 0,01$ & $\begin{array}{c}42 \pm 11 \\
*\end{array}$ & $1,23 \pm 0,30$ & $19,2 \pm 3,2$ \\
\hline & $12 \mathrm{~km} / \mathrm{h}$ & $\begin{array}{c}180 \pm 10 \\
* * *\end{array}$ & $\begin{array}{c}3,4 \pm 0,7 \\
* * *\end{array}$ & $164 \pm 7$ & $1,23 \pm 0,06$ & $0,159 \pm 0,020$ & $0,209 \pm 0,027$ & $0,53 \pm 0,07$ & $17,1 \pm 2,0$ & $74,5 \pm 2,0$ & $0,11 \pm 0,01$ & $\begin{array}{l}49 \pm 15 \\
* *\end{array}$ & $1,36 \pm 0,39$ & $20,7 \pm 3,6$ \\
\hline & VMA & $193 \pm 7$ & $7,3 \pm 1,6+$ & $170 \pm 9$ & 0,09 & 0,028 & $0,209=$ & $\underset{* *}{0,58 \pm 0,11}$ & $\underset{* * *}{14,3 \pm 1,8}$ & $\begin{array}{c}73,0 \pm 3,5 \\
* *\end{array}$ & $0,01^{*}$ & $61 \pm 27$ & 1,52 & 2 \\
\hline \multirow{4}{*}{ Tous } & $8 \mathrm{~km} / \mathrm{h}$ & $140 \pm 16$ & $1,5 \pm 0,6$ & $153 \pm 16$ & $0,88 \pm 0,12$ & $0,186 \pm 0,031$ & $0,211 \pm 0,039$ & $0,41 \pm 0,07$ & $24,8 \pm 3,6$ & $77,4 \pm 2,1$ & $0,13 \pm 0,05$ & $34 \pm 9$ & $1,15 \pm 0,24$ & $17,7 \pm 2,0$ \\
\hline & $10 \mathrm{~km} / \mathrm{h}$ & $154 \pm 17$ & $1,9 \pm 0,8$ & $160 \pm 8$ & $1,04 \pm 0,05$ & $0,172 \pm 0,015$ & $0,203 \pm 0,018$ & $0,48 \pm 0,04$ & $19,8 \pm 1,6$ & $75,6 \pm 1,2$ & $0,12 \pm 0,01$ & $38 \pm 8$ & $1,20 \pm 0,21$ & $19,2 \pm 2,3$ \\
\hline & $12 \mathrm{~km} / \mathrm{h}$ & $168 \pm 17$ & $2,4 \pm 1,1$ & $164 \pm 8$ & $1,22 \pm 0,06$ & $0,162 \pm 0,015$ & $0,205 \pm 0,019$ & $0,54 \pm 0,05$ & $16,8 \pm 1,5$ & $73,9 \pm 1,5$ & $0,11 \pm 0,01$ & $43 \pm 11$ & $1,28 \pm 0,26$ & $20,3 \pm 2,5$ \\
\hline & VMA & $195 \pm 8$ & $7,8 \pm 1,7$ & $176 \pm 12$ & $1,59 \pm 0,17$ & $0,141 \pm 0,022$ & $0,201 \pm 0,022$ & $0,66 \pm 0,12$ & $12,2 \pm 2,3$ & $70,8 \pm 3,4$ & $0,10 \pm 0,01$ & $57 \pm 20$ & $1,47 \pm 0,40$ & $22,0 \pm 2,9$ \\
\hline \multicolumn{2}{|c|}{ Influence Vitesse } & ,001 & $p<0,000$ & $p<0,001$ & $p<0,001$ & $p<0,001$ & NS & $p<0,001$ & $p<0,001$ & $0<0,001$ & $p<0,001$ & $p<0,001$ & $p<0,001$ & $<0,001$ \\
\hline
\end{tabular}

d) Influence de la fatigue

Sur base des études préliminaires, tous les paramètres biomécaniques à l'exception de IR ont été retenus pour étudier l'influence de la fatigue sur la foulée. Lors de l'épreuve sur piste, les sujets ont parcouru en moyenne $5038 \pm 2825$ m et ils ont maintenu leur effort durant $1177 \pm 510$ s. La vitesse moyenne de course était de $15,3 \pm 1,8 \mathrm{~km} / \mathrm{h}$. Le tableau 3 reprend pour l'ensemble des coureur, mais aussi pour les deux sous-populations l'évolution des paramètres biomécaniques du MyotestRun à 
trois moments clés de l'épreuve : après le premier tour (Début) au milieu de l'épreuve (Milieu) et lors du dernier enregistrement effectué avant l'épuisement du sujet (Fin). L'évolution de la fréquence cardiaque est également mesurée. L'analyse statistique pour l'ensemble de la population montre que plusieurs paramètres évoluent significativement au cours de l'épreuve. On observe une augmentation de FC, TA et DA alors que les paramètres TV, AD APJ, R, REA et $F$ se réduisent au cours de l'effort. L'analyse des sous-groupes montre que les paramètres biomécaniques présentent des évolutions significatives que dans le groupe COUREURS.

Tableau 3 : évolution pour chaque groupe des paramètres mesurés au début, au milieu et à la fin de l'épreuve réalisée à $90 \%$ de la VMA jusqu'à l'épuisement. L'influence de la fatigue sur chaque paramètre à l'intérieur de chaque groupe (Coureurs et Contrôle) et pour l'ensemble de la population (Tous) est représentée par la p value.

\begin{tabular}{|c|c|c|c|c|c|c|c|c|c|c|c|c|c|}
\hline & & $\begin{array}{c}F C \\
(b p m)\end{array}$ & $\begin{array}{c}F R \\
\text { (pas } / \text { min) }\end{array}$ & $\begin{array}{l}L G \\
(m)\end{array}$ & $\begin{array}{l}T A \\
(s)\end{array}$ & $\begin{array}{l}T V \\
(s)\end{array}$ & $\begin{array}{l}D A \\
(s)\end{array}$ & $\begin{array}{l}A D \\
\left({ }^{\circ}\right)\end{array}$ & $\begin{array}{l}A P J \\
\left({ }^{\circ}\right)\end{array}$ & $\begin{array}{l}D V \\
(m)\end{array}$ & $\begin{array}{c}R \\
(k N / m)\end{array}$ & $R E A$ & $\begin{array}{c}F \\
(N / k g)\end{array}$ \\
\hline & Début & $94 \pm 14$ & $175 \pm 10$ & $1,58 \pm 0,10$ & $0,139 \pm 0,013$ & $0,204 \pm 0,015$ & $0,064 \pm 0,006$ & $12,4 \pm 1,1$ & $70,6 \pm 1,7$ & $0,103 \pm 0,011$ & $51 \pm 10$ & $1,49 \pm 0,19$ & $23,1 \pm 2,4$ \\
\hline \multirow[t]{2}{*}{ Coureurs } & Milieu & $183 \pm 10$ & $173 \pm 9$ & $1,60 \pm 0,10$ & $0,147 \pm 0,014$ & $0,201 \pm 0,013$ & $0,068 \pm 0,007$ & $12,2 \pm 1,1$ & $69,5 \pm 1,8$ & $0,104 \pm 0,009$ & $47 \pm 9$ & $1,39 \pm 0,20$ & $22,3 \pm 2,7$ \\
\hline & Fin & $189 \pm 9$ & $174 \pm 7$ & $1,59 \pm 0,09$ & $0,149 \pm 0,014$ & $0,197 \pm 0,012$ & $0,068 \pm 0,007$ & $12,0 \pm 1,0$ & $69,3 \pm 2,0$ & $0,102 \pm 0,008$ & $46 \pm 9$ & $1,36 \pm 0,20$ & $21,8 \pm 2,8$ \\
\hline \multicolumn{2}{|c|}{ Influence fatigue } & $p<0,001$ & NS & NS & $p<0,005$ & $p<0,005$ & $\mathrm{P}<0,001$ & $p<0,01$ & $p<0,001$ & NS & $p<0,005$ & $p<0,001$ & $p<0,001$ \\
\hline \multirow{3}{*}{ Contrôle } & Début & $107 \pm 11$ & $167 \pm 7$ & $1,32 \pm 0,04$ & $0,162 \pm 0,009$ & $0,199 \pm 0,016$ & $0,059 \pm 0,004$ & $14,9 \pm 1,3$ & $72,4 \pm 1,3$ & $0,108 \pm 0,010$ & $47 \pm 9$ & $1,23 \pm 0,15$ & $20,9 \pm 1,4$ \\
\hline & Milieu & $182 \pm 5$ & $167 \pm 7$ & $1,32 \pm 0,06$ & $0,161 \pm 0,014$ & $0,200 \pm 0,016$ & $0,059 \pm 0,005$ & $15,0 \pm 1,3$ & $72,5 \pm 1,7$ & $0,108 \pm 0,010$ & $47 \pm 11$ & $1,28 \pm 0,22$ & $21,1 \pm 2,2$ \\
\hline & Fin & $187 \pm 6$ & $167 \pm 7$ & $1,32 \pm 0,06$ & $0,162 \pm 0,013$ & $0,197 \pm 0,015$ & $0,059 \pm 0,005$ & $14,8 \pm 1,2$ & $72,4 \pm 1,6$ & $0,107 \pm 0,010$ & $47 \pm 10$ & $1,23 \pm 0,20$ & $20,7 \pm 1,9$ \\
\hline \multicolumn{2}{|c|}{ Influence fatigue } & $p<0,001$ & NS & NS & NS & NS & NS & NS & NS & NS & NS & NS & NS \\
\hline \multirow{3}{*}{ Tous } & Début & $99 \pm 15$ & $172 \pm 10$ & $1,48 \pm 0,15$ & $0,148 \pm 0,016$ & $0,202 \pm 0,015$ & $0,062 \pm 0,006$ & $13,4 \pm 1,7$ & $71,3 \pm 1,8$ & $0,105 \pm 0,011$ & $49 \pm 10$ & $1,39 \pm 0,22$ & $22,2 \pm 2,4$ \\
\hline & Milieu & $183 \pm 8$ & $170 \pm 9$ & $1,50 \pm 0,16$ & $0,152 \pm 0,015$ & $0,201 \pm 0,014$ & $0,064 \pm 0,008$ & $13,3 \pm 1,8$ & $70,6 \pm 2,3$ & $0,106 \pm 0,010$ & $47 \pm 10$ & $1,35 \pm 0,22$ & $21,9 \pm 2,5$ \\
\hline & Fin & $188 \pm 8$ & $171 \pm 8$ & $1,49 \pm 0,16$ & $0,154 \pm 0,015$ & $0,197 \pm 0,013$ & $0,065 \pm 0,008$ & $13,1 \pm 1,7$ & $70,5 \pm 2,4$ & $0,104 \pm 0,009$ & $47 \pm 9$ & $1,31 \pm 0,20$ & $21,4 \pm 2,5$ \\
\hline \multicolumn{2}{|c|}{ Influence fatigue } & $p<0,001$ & NS & NS & $p<0,005$ & $p<0,005$ & $P<0,005$ & $p<0,005$ & $p<0,001$ & NS & $\mathrm{p}<0,01$ & $p<0,001$ & $\mathrm{p}<0,001$ \\
\hline
\end{tabular}

\section{Discussion}

L'utilisation du MyotestRun dans l'analyse de la course à pied est relativement récente et les quelques publications qui ont étudié la qualité de cet outil de terrain montrent des résultats contradictoires. Ainsi l'étude de Goutebarge et al[16] compare le MyotestRun avec un accéléromètre validé placé sur la chaussure et montre que FR est un paramètre valide et reproductible, au contraire de TA. L'étude montre une sous-estimation systématique du temps de contact au sol par le MyotestRun. Si ce biais systématique est confirmé par d'autres travaux [10,23], ce n'est par contre pas le cas pour la reproductibilité du paramètre TA qui apparaît excellente dans l'étude de Gindre et al[10]. Nos résultats vont plutôt dans ce sens puisque nous observons pour tous les paramètres, à l'exception d'IR, une excellente reproductibilité intra-séance du MyotestRun. Ces observations sont valables aussi bien à $10 \mathrm{~km} / \mathrm{h}$ qu'à $90 \%$ de la VMA. Dans notre étude ainsi que dans celle de Gindre et al [10], l'analyse de la foulée a été réalisée dans une ligne droite conformément aux recommandations des constructeurs alors que l'enregistrement dans l'étude de Goutebarge et al [17] s'est réalisé sur un tour complet d'une piste d'athlétisme incluant de la course en virage. Par ailleurs, il apparait que le MyotestRun était positionné à la hanche et non sous l'ombilic. Ces différences méthodologiques expliquent certainement les mauvais résultats observés pour TA et afin de garantir des mesures de qualité il est indispensable que les mesures se fassent exclusivement en ligne droite, à allure constante avec un MyotestRun positionné dans le respect des recommandations du constructeur.

Le protocole de recherche mis sur pied a permis de comparer à une même allure la course réalisée sur tapis roulant avec la course réalisée sur piste. L'analyse des résultats montre qu'il n'y a aucune différence significative entre les deux situations. Ces résultats sont conformes aux travaux de van Ingen Schenau qui montre par modélisation mathématique qu'il n'y aurait pas de différence entre 
les deux courses à l'exception de la résistance à l'air qui est inexistante sur tapis roulant [24 ]. Plusieurs études soulignent une légère augmentation de la fréquence sur tapis roulant $[25,26]$. Cette augmentation, également constatée n'atteint pas la signification statistique dans notre étude. Selon Riley et al [26 ] à partir du moment où les sujets sont familiarisés avec la course sur tapis roulant et que celui-ci est suffisamment rigide et la vitesse bien régularisée, la course sur tapis roulant peut être utilisée pour analyser la foulée d'un individu. La reproductibilité des paramètres biomécaniques du MyotestRun entre la course sur tapis roulant et la course sur piste est moins bonne qu'en condition inter-essais tout en restant satisfaisante ( $C V<10 \%$ et des $\mathrm{CCl}$ qui varient entre 0,63 et 0,82 ). Cette plus grande variabilité s'explique facilement par les différents facteurs pouvant altérer les mesures réalisées : le contexte de course (tapis roulant versus sol), l'installation du matériel qui peut différer quelque peu d'une fois à l'autre, les conditions atmosphériques qui ne sont pas standardisées à l'extérieur ou encore les différences individuelles de forme, de sensation ou de comportement d'une séance à l'autre.

La sensibilité d'un outil peut être appréciée par sa capacité à détecter de manière significative des petites modifications lors des changements de comportements. C'est ce qui a été constaté, lorsque nous avons étudié l'évolution des paramètres biomécaniques du MyotestRun avec l'augmentation de la vitesse. L'analyse des études montre une augmentation hautement significative pour FR, LG, DA, R, REA et F; une diminution pour TA, AD, APJ, DV et stagnation de TV. Nos résultats s'accordent pour la plupart avec les études qui ont étudié l'évolution des paramètres biomécaniques de la foulée avec l'augmentation de la vitesse [16,19,27-29 ]. Les mesures de fréquence et de longueur d'appuis sont conformes à la littérature [27 ], par contre il apparaît des différences en ce qui concerne TA et TV. Les études ont clairement montré que le MyotestRun diminue TA et augmente TV en comparaison avec des outils de références [10,17,23 ]. L'explication se trouverait dans le système d'identification des différentes phases d'appui utilisé par le MyotestRun qui se base sur la mesure du temps de contact effectif et non le temps de contact réel. Le temps de contact effectif correspond à la durée de l'appui durant laquelle la composante verticale de la force de réaction du sol est supérieure ou égale au poids du coureur [16 ]. La plupart des études analyse le temps de contact réel et donc s'accompagne d'un temps de contact plus court et d'un temps de vol plus long. Cette différence est également à l'origine du comportement différent observé pour le TV qui n'augmente pas avec la vitesse dans notre étude alors qu'il augmente avec la vitesse dans l'étude de Millet et al [29 ]. Cette différence de comportement est également mise en évidence dans l'étude de Gindre et al [10 ] qui compare le MyotestRun à l'Optojump et à un une caméra haute vitesse. Les auteurs proposent des équations permettant de convertir le temps de contact effectif obtenu à partir du MyotestRun en temps de contact réel tel qu'il est obtenu à partir d'un Optojump ou d'une caméra haute vitesse [10 ] L'analyse de la littérature montre que la diminution du déplacement vertical avec l'augmentation de la vitesse est conforme à la littérature [16,29,30 ]. L'évolution de APJ [30 ] mais aussi de $\mathrm{R}$ [31] sont également en accord avec la littérature et si les comparaisons de résultats sont impossibles étant donné les différences de protocoles, les valeurs du MyotestRun apparaissent assez proches de celles observées dans ces études pour des niveaux de vitesse comparables.

L'ensemble des résultats de ces trois analyses préliminaires tendent à montrer que le 
MyotestRun présente les qualités suffisantes pour pouvoir analyser l'impact de la fatigue sur les paramètres biomécaniques du coureur. Tous les paramètres sélectionnés, à l'exception de l'index de régularité semblent pouvoir être conservés dans l'analyse.

L'objectif principal de notre étude était d'analyser l'influence de la fatigue sur les paramètres biomécaniques de la foulée lors d'une épreuve réalisée à $90 \%$ de la VMA jusqu'à l'épuisement. La durée de l'effort maintenu par nos sujets $(1177 \pm 510$ s) apparaît plus élevée que celles observées dans les études ayant couplé une mesure des gaz respiratoires à une épreuve de temps limite à $90 \%$ de VMA [32-35]. Les conditions expérimentales différentes pourraient partiellement expliquer ces différences. Nos résultats par contre concordent parfaitement avec la modélisation mathématique proposée par Bosquet et al [36]. Ce résultat, ainsi que les valeurs de fréquence cardiaque (96\%Fmax) très proches du maximum mesure lors de l'épreuve progressive sur tapis roulant, confirment la bonne collaboration des participants.

L'analyse des paramètres biomécaniques confirme l'impact significatif de la fatigue sur la foulée. L'augmentation du temps d'appui (TA) avec la fatigue est conforme à ce que l'on retrouve dans la plupart des études de ce type [20,21,35,37-39]. L'analyse de l'ensemble des paramètres mesurés par le MyotestRun montre que cette plus longue durée des appuis s'accompagne d'une plus grande distance parcourue sur l'appui, et parallèlement d'une diminution de TV, de APJ, de AD, de REA, de R et de F. Par contre il n'y a aucune modification significative de DV. Si la plupart de ces résultats sont globalement conformes aux études précédentes [18,21,35,37,38,40-42], quelques différences apparaissent. Elliot et Ackland avaient déjà montré en 1981 que le déplacement horizontal du bassin au-dessus de l'appui était augmenté avec la foulée [18] alors que Rabita et al [21] observent des modifications comparables de APJ et de AD lors d'une épreuve réalisée à $95 \%$ de la VMA. Plusieurs auteurs observent une diminution de la composante verticale de la force à l'appui qui résulterait d'une diminution de la force des muscles du membre inférieur avec comme conséquence une impulsion au sol moins intense et plus amortie. Seule l'étude de Fourchet et al [38] montre une augmentation de la force. Les auteurs justifient ce résultat contradictoire par le jeune âge des coureurs (moyenne de 16,9 ans) et un manque d'expérience dans la gestion de la fatigue lors d'un effort prolongé en course à pied. L'augmentation du déplacement vertical du centre de masse avec la fatigue pourrait expliquer une majoration de la composante verticale de la force appliquée au sol. Ces adolescents Le jeune âge et la manque d'expérience des sujets de cette étude pourraient justifier ce résultat contradictoire. D'après les travaux de Morin et al [43], à vitesse constante, la raideur verticale est hautement influencée par la durée du temps d'appui. Conformément à cette étude, mais aussi aux travaux de Dutto et Smith [44], Girard et al [37], Hunter et al [40] Slawinski et al [42], l'augmentation de TA s'accompagne d'une diminution de la raideur verticale. Rabita et al [21] n'observent aucune modification de ce paramètre alors qu'ils constatent une diminution de la raideur du membre inférieur. La raideur verticale prend en compte l'oscillation verticale du corps et alors que la raideur du membre inférieur prend en considération la variation de longueur du membre inférieur à l'amortissement. Ce paramètre pourrait s'avérer plus sensible que la raideur verticale, mais n'est pas actuellement mesuré par le MyotestRun. 

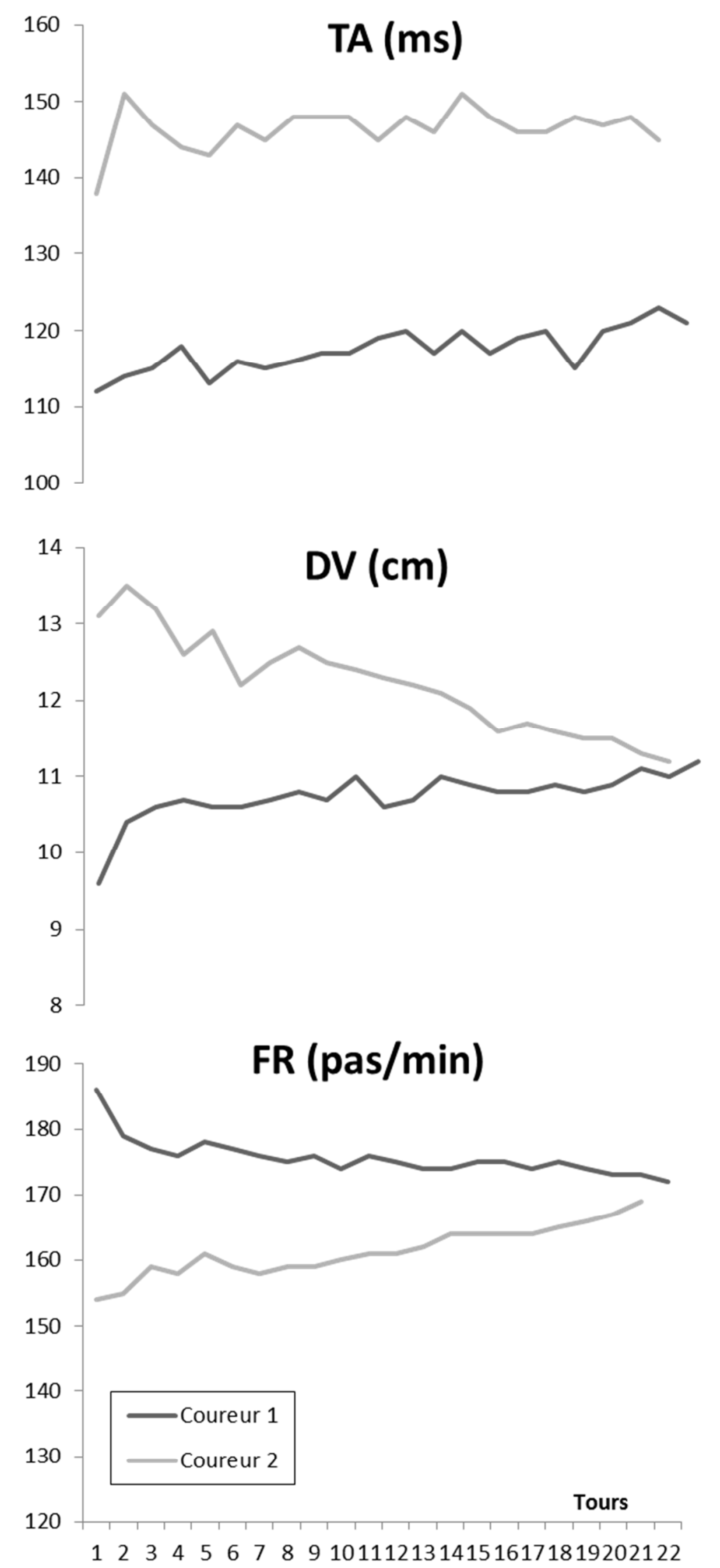

Figure 1 : représentation graphique de l'évolution de TA, DV et FR à chaque tour de l'épreuve réalisée à $90 \%$ de la VMA observée auprès de deux sujets différents (coureur 1 et coureur 2). L'augmentation progressive de TA chez le coureur 1 s'accompagne d'une augmentation de la fréquence et d'une diminution de DV, alors que chez le coureur 2 on observe le phénomène inverse. 
L'évolution de la FR avec la fatigue apparaît très contradictoire. Si la plupart des recherches montrent une réduction significative de ce paramètre [19,20,35,37,39,40,44,45] quelques-unes comme la nôtre ne montrent aucune évolution $[18,38,41]$ et d'autres observent une augmentation de cette fréquence $[21,29,46]$. Ces différences observées d'une étude à l'autre pourraient être influencées par : 1) la durée et l'intensité de la course ; 2) le type d'épreuve (temps limite à intensité constante ou chronomètre sur une distance donnée) ; 3) le niveau d'expérience du coureur ; 4) les conditions dans lesquelles les efforts ont été réalisés (tapis roulant versus piste ou route). Si des études complémentaires apparaissent nécessaires pour clarifier l'influence de ces différents facteurs sur l'évolution des paramètres biomécaniques de la foulées avec la fatigue, un grand nombre de publications souligne l'importante variabilité interindividuelle [20,40,44,47,48]. Une analyse plus approfondie de nos résultats montre effectivement que les adaptations de la foulée avec l'apparition de la fatigue ne sont pas les mêmes chez tous les sujets. Certains comportements semblent même antagonistes. A titre d'exemple, lorsque l'on étudie l'évolution de la fréquence tout au long de l'épreuve, on n'observe aucune variation significative sur l'ensemble de nos coureurs. Une analyse au cas par cas montre qu'il existe en réalité des réponses très différentes avec certains sujets qui augmentent progressivement la fréquence alors que d'autres la diminuent. La Figure 1 montre ainsi l'exemple de deux sujets présentant des patterns de fatigue différents avec notamment des évolutions différentes de FR, TA et DV. Ces résultats confirment que dès le départ il existe des modèles de foulée individuels, mais également que cette foulée peut évoluer de différente manière avec l'apparition de la fatigue. Les résultats moyens observés dans une population offrent certes une tendance générale mais cachent en réalité cette diversité. Une plus grande diversité des patterns de fatigue au sein du groupe CONTROLE pourrait expliquer qu'aucune évolution significative des paramètres biomécaniques ne ressort en comparaison avec le groupe COUREURS. Une plus grande variabilité des paramètres biomécaniques chez les coureurs moins expérimentés a d'ailleurs été soulignée par Slawinski et al [41].

Plusieurs recherches ont tenté d'identifier les différents types de foulée rencontrés chez les coureurs. L'étude de Gindre et al [49] identifie deux grandes catégories de coureurs : les terriens et les aériens. Le terriens présentent un temps de contact au sol et une compression de la jambe plus importante alors que les aériens se caractérisent pas un plus long temps de vol, une oscillation verticale plus importante; une plus grande force au sol, et une plus grande raideur. Etant donné les différences importantes observées dès le départ entre les coureurs, il n'est pas surprenant d'observer des évolutions différentes des patterns de course avec la fatigue. Une approche de l'analyse de la foulée et de l'impact de la fatigue plus individuelle ou par catégorie semble se justifier au regard des connaissances actuelles. Un des objectifs des recherches futures sera d'étudier s'il existe un lien entre le type de course et le type de fatigue. Un autre objectif sera d'étudier s'il existe des stratégies optimales de modification de la foulée avec la fatigue et si, éventuellement, la stratégie à adopter pourrait être dépendante du type de course de l'athlète. De nombreuses perspectives de recherche existent dans ce domaine et au regard des résultats obtenus dans cette étude le MyotestRun apparaît comme un outil offrant de réelles perspectives d'analyses biomécaniques et de recherche sur le terrain tout en restant accessible à de nombreux entraîneurs. 


\section{Conclusion}

L'analyse préliminaire démontre que le MyotestRun apparaît comme un outil intéressant présentant un niveau de reproductibilité et de sensibilité satisfaisant autorisant son utilisation dans le cadre de recherches appliquées sur le terrain. Si les problèmes de validité concurrente mis en évidence par certains auteurs limitent les possibilités de comparaison des résultats, il apparaît que l'outil est parfaitement capable de mettre en évidence l'évolution de la foulée en fonction de la vitesse ou encore avec la fatigue. L'analyse de l'influence de la fatigue sur la foulée s'accorde avec la plupart des études, et confirme surtout qu'il existe des différences interindividuelles très importantes. Les recherches futures devront étudier de manière plus approfondie les différents styles de course ainsi que les relations entre les styles de course et l'évolution des paramètres biomécaniques avec la fatigue.

\section{Références}

1. Kyr H, Inen L, Belli A, Komi P V. Biomechanical factors affecting running economy. Med Sci Sport Exerc . 2001 ;33(8):1330-7.

2. Anderson T. Biomechanics and running economy. Sports Med . 1996 Aug ;22(2):76-89.

3. Nummela A, Keränen T, Mikkelsson L. Factors Related to Top Running Speed and Economy. Int J Sports Med . 2007 Aug $1 ; 28(8): 655-61$.

4. Altman AR, Davis IS. Barefoot Running. Curr Sports Med Rep . $2012 ; 11(5): 244-50$.

5. Foch E, Reinbolt JA, Zhang S, Fitzhugh EC, Milner CE. Associations between iliotibial band injury status and running biomechanics in women. Gait Posture . $2015 \mathrm{Feb} ; 41(2): 706-10$.

6. McMahon TA, Valiant G, Frederick EC. Groucho running. J Appl Physiol . 1987 ;62(6).

7. Fyhrie DP, Milgrom C, Hoshaw SJ, Simkin A, Dar S, Drumb D, et al. Effect of Fatiguing Exercise on Longitudinal Bone Strain as Related to Stress Fracture in Humans. Ann Biomed Eng . 1998 Jul :26(4):660-5.

8. Watari, R., Hettinga, B., Osis, S., \& Ferber, R. Validation of a Torso-Mounted Accelerometer for Measures of Vertical Oscillation and Ground Contact Time During Treadmill Running. J Appl Biomechanics, 2015;11(1), 86-95.

9. Laffaye G, Jidovsteff B. Analyse des sauts et de la course à pied par accélérométrie. Instrum Mes Metrol. 2014;14(34):229-49.

10. Gindre C, Lussiana T, Hebert-Losier K, Morin J-B. Reliability and validity of the Myotest $\circledast$ for measuring running stride kinematics. J Sports Sci . 2016 Apr $2 ; 34(7): 664-70$.

11. Bergamin M, Ermolao A, Sieverdes JC, Zaccaria M, Zanuso S. Validation of the mywellness key in walking and running speeds. J Sports Sci Med . $2012 ; 11(1): 57-63$.

12. Norris M, Anderson R, Kenny IC. Method analysis of accelerometers and gyroscopes in running gait: A systematic review. Proc Inst Mech Eng Part P J Sport Eng Technol. 2014 Mar 16 ;228(1):3-15.

13. Hausswirth C, Le Meur Y, Couturier A, Bernard T, Brisswalter J. Accuracy and Repeatability of the Polar ? RS800sd to Evaluate Stride Rate and Running Speed. Int J Sports Med. 2009 May 10 ;30(5):354-9.

14. Hunter I, Tracy J, Walker S, Eatough J. Accuracy of running data from the garmin forerunner. ; Available from: http://archive.asbweb.org/conferences/2015/abstracts/64BD--Accuracy Of Running Data From The Garmin Forerunner--(Hunter).pdf

15. Balsalobre-Fernandez C, Agopyan H, Morin J-B. The Validity and Reliability of an iPhone App for Measuring Running Mechanics. J Appl Biomech . 2017 Jun $26 ; 1-5$.

16. Cavagna GA, Franzetti $P$, Heglund NC, Willems $P$. The determinants of the step frequency in running, trotting and hopping in man and other vertebrates. J Physiol . 1988 May 1 ;399(1):81-92. Available from: http://doi.wiley.com/10.1113/jphysiol.1988.sp017069

17. Gouttebarge V, Wolfard R, Griek N, Ruiter CJ de, Boschman JS, Dieën JH van. Reproducibility and Validity of the Myotest for Measuring Step Frequency and Ground Contact Time in Recreational Runners. J Hum Kinet . 2015 Jan 1 ;45(1):19-26.

18. Elliot B, Ackland T. Biomechanical Effects of Fatigue on 10,000 Meter Running Technique. Res Q Exerc Sport . 1981 May ;52(2):160-6.

19. Candau R, Belli A, Millet GY, Georges D, Barbier B, Rouillon JD. Energy cost and running mechanics during a treadmill run to voluntary exhaustion in humans. Eur J Appl Physiol . 1998 May $1 ; 77(6): 479-85$.

20. Gazeau F, Koralsztein J, Billat V. Biomechanical events in the time to exhaustion at maximum aerobic speed. Arch Physiol . 1997 ; 105(6):583-590.

21. Rabita G, Slawinski J, Girard O, Bignet F. Spring-mass behavior during exhaustive run at constant velocity in elite triathletes. Med Sci . 2011;43(4):685-692.

22. Portney LG, Watkins MP, Portney G WP. Foundations of Clinical Research: Applications to Practice. New Jersey: Pearson Education,Inc. 2009. 892 p.

23. Deflandre D, Schwartz C, Weerts JP, Croisier J-L, Bury T. A Comparison of 3D Methods for Identifying the Stance 
Phase in Treadmill Running for Both Rearfoot and Forefoot Runners. J Sport Sci . 2016 Jun 28 ;4(3):124-31.

24. van Ingen Schenau GJ. Some fundamental aspects of the biomechanics of overground versus treadmill locomotion. Med Sci Sports Exerc . $1980 ; 12(4): 257-61$.

25. Elliot BC, Blanksby BA. A cinematographic analysis of overground and treadmill running by males and females. Med Sci Sport Exerc. 1976;8(2):84-87.

26. Riley PO, Dicharry J, Franz J, Croce U Della, Wilder RP, Kerrigan DC. A Kinematics and Kinetic Comparison of Overground and Treadmill Running. Med Sci Sport Exerc. $2008 ; 40(6): 1093-100$.

27. Cavanagh $P$, Kram R. Stride length in distance running: velocity, body dimensions, and added mass effects. Med Sci Sport Exerc . $1989 ; 21(4): 467-79$.

28. Leboeuf $F$, Achard De Leluardière $F$, Lacouture $P$, Duboy J, Leplanquais $F$, Junqua A. Étude biomécanique de la course. Encyclopédie Médico-chirurgicale, traité de podologie. 2006;1-16. 28.

29. Millet GY, Morin J-B, Degache F, Edouard P, Feasson L, Verney J, et al. Running from Paris to Beijing: biomechanical and physiological consequences. Eur J Appl Physiol . 2009 Dec 16 ;107(6):731-8.

30. Lee CR, Farley CT. Determinants of the center of mass trajectory in human walking and running. J Exp Biol . 1998 ;201(21).

31. Arampatzis A, Brüggemann G, Metzler V. The effect of speed on leg stiffness and joint kinetics in human running. J Biomech . 1999;32(12):1349-1353. ;

32. Blondel N, Berthoin S, Billat V. Relationship between run times to exhaustion at 90, 100,120, and 140\% of vV O2max and velocity expressed relatively to critical velocity and maximal velocity. Int J . 2001;22(01):27-33. ;

33. Billat V, Binsse V, Petit B, Koralsztein JJP. High Level Runners Are Able to Maintain a VO2 Steady-State Below VO2max in an All-Out Run Over Their Critical Velocity. Arch Physiol Biochem . 1998 Jan 3 ;106(1):38-45.

34. Billat VL, Richard R, Binsse VM, Koralsztein JP, Haouzi P. The Vo2 slow component for severe exercise depends on type of exercise and is not correlated with time to fatigue. J Appl Physiol . 1998 ;85(6):2118-2124.

35. Avogadro P, Dolenec A, Belli A. Changes in mechanical work during severe exhausting running. Eur J Appl Physiol . 2003; 90(1-2):165-170. ;

36. Bosquet L, Léger L, Legros P. Methods to Determine Aerobic Endurance. Sport Med. 2002; 32(11):675-700. ;

37. Girard $\mathrm{O}$, Micallef $\mathrm{J}$, Millet $\mathrm{G}$. Changes in spring-mass model characteristics during repeated running sprints. Eur $\mathrm{J}$ Appl Physiol . 2011; 111(1):125-134. ;

38. Fourchet F, Girard O, Kelly L, Horobeanu C, Millet GP. Changes in leg spring behaviour, plantar loading and foot mobility magnitude induced by an exhaustive treadmill run in adolescent middle-distance runners. J Sci Med Sport . 2015 Mar ;18(2):199-203.

39. Chan-Roper M, Hunter I, W Myrer J, L Eggett D, K Seeley M. Kinematic changes during a marathon for fast and slow runners. J Sports Sci Med . $2012 ; 11(1): 77-82$.

40. Hunter I, Smith GA. Preferred and optimal stride frequency, stiffness and economy: changes with fatigue during a 1-h high-intensity run. Eur J Appl Physiol . 2007 Jul 27 ;100(6):653-61.

41. Slawinski J, Billat V. Changes in internal mechanical cost during overground running to exhaustion. Med Sci Sport Exerc . 2005;37(7):1180-1186 ;

42. Slawinski J, Heubert R, Quievre J, Billat V. Changes in spring-mass model parameters and energy cost during track running to exhaustion. J . 2008; 22(3), 930-936.;

43. Morin J-B, Dalleau G, Kyröläinen H, Jeannin T, Belli A. A Simple Method for Measuring Stiffness during Running. J Appl Biomech . 2005 May 1;21(2):167-80.

44. Dutto DJ, Smith GA. Changes in spring-mass characteristics during treadmill running to exhaustion. Med Sci Sport Exerc . 2002];34(8):1324-31.

45. Mizrahi J, Verbitsky O, Isakov E, Daily D. Effect of fatigue on leg kinematics and impact acceleration in long distance running. Hum Mov Sci . 2000;19(2):139-151. ;

46. Kyröläinen $\mathrm{H}$, Pullinen $\mathrm{T}$, Candau R, Avela J, Huttunen $\mathrm{P}$, Komi $\mathrm{P} \vee$. Effects of marathon running on running economy and kinematics. Eur J Appl Physiol . 2000 Jul 24 ;82(4):297-304.

47. Nicol C, Komi P V., Marconnet P. Fatigue effects of marathon running on neuromuscular performance. Scand J Med Sci Sports . 2007 Jan $30 ; 1(1): 10-7$.

48. Halvorsen K, Eriksson M, Gullstrand L. Acute Effects of Reducing Vertical Displacement and Step Frequency on Running Economy. J Strength Cond Res . 2012 Aug ;26(8):2065-70.

49. Gindre C, Lussiana T, Hebert-Losier K, Mourot L. Aerial and Terrestrial Patterns: A Novel Approach to Analyzing Human Running. Int J Sports Med . $2015 ; 37(1): 25-6$. 